\title{
COMPARISON BETWEEN DISK-LIKE OBJECTS FORMED IN HIERARCHICAL HYDRODYNAMICAL SIMULATIONS AND OBSERVATIONS OF SPIRAL GALAXIES
}

\author{
A. SÁIZ AND R. DOMÍNGUEZ-TENREIRO \\ Depto. de Física Teórica, Universidad Autónoma de Madrid \\ Madrid, E-28049, Spain \\ P. B. TISSERA \\ Intituto de Astronomía y Física del Espacio \\ Buenos Aires, 1428, Argentina \\ AND \\ S. COURTEAU \\ Univ. of British Columbia, Dept. of Physics and Astronomy \\ Vancouver, BC, Canada V6T $1 Z 1$
}

\begin{abstract}
We analyze the structural and dynamical properties of disk-like objects formed in fully consistent cosmological simulations which include inefficient star formation. Comparison with data of similar observable properties of spiral galaxies gives satisfactory agreement, in contrast with previous findings using other codes. This suggests that the stellar formation implementation used has allowed the formation of disks as well as guaranteed their stability.
\end{abstract}

\section{Introduction}

We present results of a detailed comparison between the parameters characterizing the structural and dynamical properties of a sample of 29 simulated disk-like objects (DLOs) and those measured in observed spiral galaxies. The properties we focus on are the bulge and disk structural parameters and the rotation curves, as they can be constrained with available data for observed spiral galaxies. These data are taken principally from the compilation of galaxy structural parameters of Broeils (1992), de Jong (1996) and Courteau (1996, 1997). 

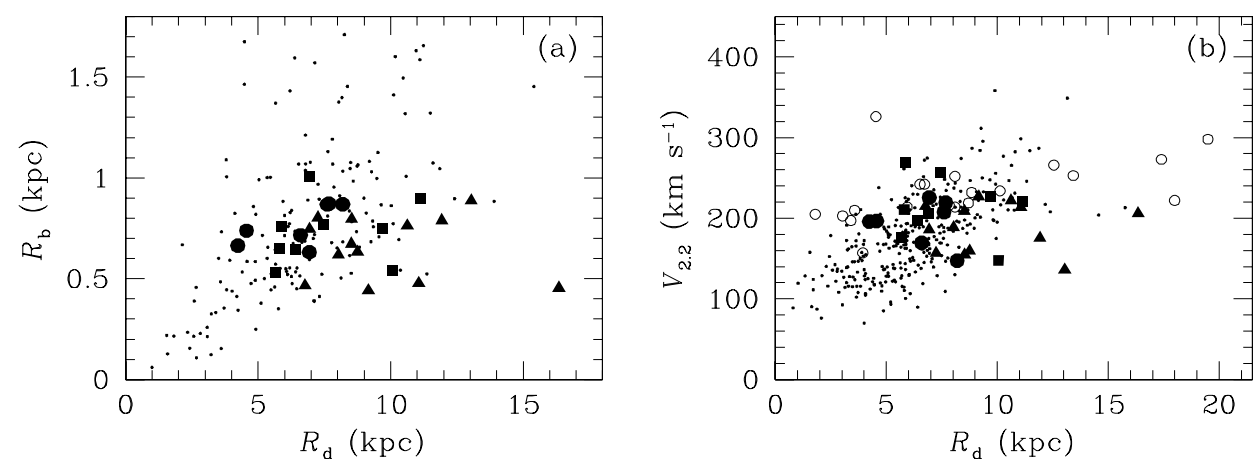

Figure 1. The scale lengths $R_{\mathrm{b}}$ and $R_{\mathrm{d}}$ (a), and the $V_{2.2}$ velocities versus $R_{\mathrm{d}}(\mathrm{b})$. Filled symbols correspond to DLOs formed in our simulations. Different symbol shapes correspond to objects formed in different realizations. Dots in (a) are from 1D decompositions of surface brightness profiles given by Courteau, de Jong, \& Broeils (1996) and Courteau (1997). Dots in (b) are data from the Courteau-Faber (H $\alpha$ spectra) sample. Open circles are data from spirals with H I rotation curves from Casertano \& van Gorkom (1991).

The DLOs have been identified in AP3M-SPH fully consistent hierarchical hydrodynamical simulations, realizations of a CDM flat model, with $\Omega_{\mathrm{b}}=0.1, \Lambda=0$ and $b=2.5$, made using $64^{3}$ particles in a periodic box of comoving side $10 \mathrm{Mpc}\left(\mathrm{H}_{0}=50 \mathrm{~km} \mathrm{~s}^{-1} \mathrm{Mpc}^{-1}\right)$, and where an inefficient Schmidt law-like algorithm to model the stellar formation processes has been implemented (see Tissera, Lambas, \& Abadi 1997; Silk 1999).

\section{Bulge-disk decomposition}

A surface density bulge-disk decomposition was performed on the DLOs, using a double-exponential profile,

$$
\Sigma(R)=\Sigma_{\mathrm{b}}(0) \exp \left[-R / R_{\mathrm{b}}\right]+\Sigma_{\mathrm{d}}(0) \exp \left[-R / R_{\mathrm{d}}\right] .
$$

The resulting bulge and disk scale lengths, $R_{\mathrm{b}}$ and $R_{\mathrm{d}}$, and their ratio $R_{\mathrm{b}} / R_{\mathrm{d}}$, are consistent with available data (Courteau, de Jong, \& Broeils 1996; de Jong 1996; Moriondo, Giovanelli, \& Haynes 1999). See Fig. 1(a).

\section{Rotation curves}

The rotation curves of the simulated objects can be constructed by adding up in quadrature the contributions to circular rotation from the bulge and the disk, both formed by baryonic particles, and the dark matter halo,

$$
V_{\text {cir }}^{2}(r)=V_{\text {bar }}^{2}(r)+V_{\mathrm{dm}}^{2}(r) .
$$

Our rotation curve analysis and shape modeling uses a parameterization based on the spatial scale $R_{2.2}=2.2 R_{\mathrm{d}}$ and the velocity (mass) estimate 
$V_{2.2}=V_{\text {cir }}\left(R_{2.2}\right)$. These parameters were compared with those measured for the Courteau-Faber sample of bright $\mathrm{Sb}$-Sc field spirals with long-slit $\mathrm{H} \alpha$ rotation curves, and the Broeils (1992) compilation of late-type spirals with extended H I rotation curves. Deep surface photometry is available for both samples. In contrast to findings in other fully consistent hydrodynamical simulations (Navarro \& Steinmetz 2000 and references therein), we find DLO $V_{2.2}$ values that are consistent with the observational data. This is a consequence of disk formation with conservation of the specific angular momentum $j$. See Fig. 1(b).

\section{Luminous and dark matter contributions}

In real spirals, the relative contributions of baryons and dark matter to the rotation curve cannot be inferred uniquely from the observations. One has to postulate some hypothesis to infer the mass distribution of the halo. The so-called 'maximum disk (or maximal light) hypothesis' (van Albada \& Sancisi 1986) assumes that the halo dark mass component needed to fit the rotation curve should be minimum, or, equivalently, that the contribution of the disk and bulge to the inner parts of the rotation curves should be maximum. Considering this, one has $\left(V_{\text {lum }} / V_{\text {cir }}\right)_{R_{2.2}} \simeq 0.85 \pm 0.10$, at the $95 \%$ confidence level (Sackett 1997). The other common constraint used is that of sub-maximal disks, obtained either by matching the vertical velocity dispersion, scale height and scale length of a thin exponential disk which yields the maximum disk rotation (Bottema 1993), or, independently, showing that the Tully-Fisher relation of bright spirals is independent of surface brightness (Courteau \& Rix 1999). Then one has $\left(V_{\text {lum }} / V_{\text {cir }}\right)_{R_{2.2}} \simeq 0.60 \pm 0.10$.

The average relative contribution of baryons to $V_{2.2}$ in our sample of DLOs can be measured directly. We find $\left(V_{\text {bar }} / V_{\text {cir }}\right)_{R_{2.2}}=0.67 \pm 0.07$, in very good agreement with Bottema $(1993,1997)$ and Courteau \& Rix (1999). See Fig. 2. Most DLOs have been found to have sub-maximal

disks. This means that the amount of baryon mass that has ended up inside $R<R_{2.2}$ is not excessive, again as a consequence of $j$ conservation.

\section{Conclusions}

In conclusion, the comparison between the DLOs produced in our simulations and observational data allows us to affirm that the DLOs have counterparts in today spiral galaxies. This agreement suggests that the process operating in Fall \& Efstathiou (1980) standard model for disk formation (i.e., gas cooling and collapse with $j$ conservation) is also at work in the quiescent phases of the DLO formation in these simulations. However, violent episodes (i.e., interactions and merger events) also occur and play an important role in the DLO assembly (Domínguez-Tenreiro, Tissera, \& Sáiz 1998; 


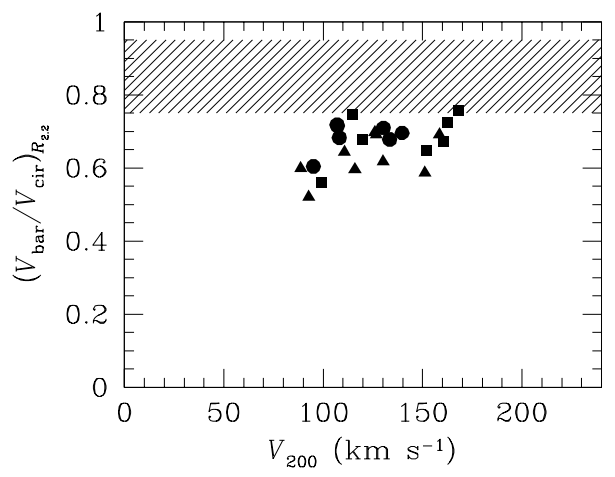

Figure 2. The $\left(V_{\mathrm{bar}} / V_{\text {cir }}\right)_{R_{2.2}}$ ratios versus the circular velocities at virial radius, $V_{200}$, for the DLO sample. Different symbol shapes correspond to objects formed in different realizations. The shaded area shows the $95 \%$ confidence interval for maximum disks.

Sáiz, Tissera, \& Domínguez-Tenreiro 1999). To provide the right conditions for disk regeneration after the last violent episode of the DLO assembly, a compact central stellar bulge is needed; this will ensure the axisymmetric character of the gravitational potential well at scales of some kpcs at all times, avoiding excessive $j$ losses in violent events. A second condition is necessary: the availability of gas at low $z$, to form the disk. The good match of our DLO parameters with observational data suggests that our inefficient star formation algorithm meets both requirements. This global agreement with observations also represents an important step towards making numerical approaches more widely used for the study of galaxy formation and evolution in a cosmological framework, i.e., from primordial fluctuations.

\section{References}

Bottema R., 1993, A\&A, 275, 16; 1997, A\&A, 328, 517

Broeils A. H., 1992, Ph.D. thesis, Univ. Groningen

Casertano S., van Gorkom J. H., 1991, AJ, 101, 1231

Courteau S., 1996, ApJS, 103, 363; 1997, AJ, 114, 2402

Courteau S., Rix H.-W., 1999, ApJ, 513, 561

Courteau S., de Jong R. S., Broeils A.H., 1996, ApJ, 457, L73

de Jong R. S., 1996, A\&A, 313, 45

Domínguez-Tenreiro R., Tissera P. B., Sáiz A., 1998, ApJ, 508, L123

Fall S. M., Efstathiou G., 1980, MNRAS, 193, 189

Moriondo G., Giovanelli R., Haynes M. P., 1999, A\&A, 346, 415

Navarro J. F., Steinmetz M., 2000, ApJ, 538, 477

Sackett P. D., 1997, ApJ, 483, 103

Sáiz A., Tissera P. B., Domínguez-Tenreiro R., 1998, Ap\&SS, 263, 43

Silk J., 1999, in Proceedings of the XIXth Moriond Meeting, eds. F. Hammer, T. X.

Thuan, V. Cayatte, B. Guiderdoni, \& J. Tran Thanh Van, Ed. Frontières, 439

Tissera P. B., Lambas D. G., Abadi M. G., 1997, MNRAS, 286, 384

van Albada T. S., Sancisi R., 1986, Phil. Trans. R. Soc. London Ser. A, 320, 447 